\title{
ESTRATÉGIAS APRISIONAMENTO DE GASES RESIDUAIS PARA MOTORES DE IGNIÇÃO POR CENTELHA COM ETANOL DE ALTA EFICIÊNCIA
}

\author{
Thompson D. M. Lanzanova ${ }^{1}$, Maria F. P. Mazer ${ }^{1}$, \\ Leonardo Hatschbach ${ }^{1}$, Hua Zhao ${ }^{2}$ \\ ${ }^{1}$ Universidade Federal de Santa Maria, ${ }^{2}$ Brunel University London \\ E-mails: lanzanova@gmail.com
}

\begin{abstract}
RESUMO
Os biocombustíveis vêm sendo explorados na tentativa de reduzir as emissões de gases do efeito estufa e o consumo de combustíveis fósseis. Por esses mesmos propósitos, as recentes diretrizes legais sobre o controle de emissões demandam maiores eficiências para motores de ignição por centelha (SI) operando em carga parcial. Nesse contexto, este estudo investigou a utilização de estratégias não convencionais de tempos de válvula em um motor de testes monocilíndrico de 0,35 L e quatro válvulas operando com etanol anidro. Esse era equipado com um sistema de atuação de válvulas independente que permitia variabilidade total de momentos de abertura e fechamento de válvulas. Os processos negative valve overlap (NVO) e exhaust rebreathing (ER) foram avaliados como estratégias de aprisionamento de gases residuais. $\mathrm{O}$ fechamento precoce das válvulas de admissão (early intake valve closure, EIVC) foi empregado como método de controle de carga. A combustão SI convencional, com controle de carga através de restrição por borboleta na admissão (throtle spark ignition, $t S I$ ), foi testada para fins de comparação. Várias cargas parciais foram avaliadas em rotação fixa de $1500 \mathrm{rpm}$. O aumento de eficiência indicada pôde ser alcançado ao aplicar as estratégias de NVO e ER.
\end{abstract}

\section{INTRODUÇÃO}

$\mathrm{O}$ aumento de eficiência dos motores representa um dos maiores objetos de pesquisa na busca por redução de emissões de gases do efeito estufa. Estima-se que cerca de $20 \%$ a $30 \%$ do $\mathrm{CO}_{2}$ produzido nos países desenvolvidos é proveniente do setor de transportes [1], [2]. Para reduzir as emissões geradas por veículos leves, legislações severas estão limitando gradativamente a quantidade de $\mathrm{CO}_{2} / \mathrm{km}$ liberado, exigindo assim a aplicação de tecnologias avançadas para aumentar a eficiência dos motores. No cenário atual, também é conveniente que muitos países que não produzem petróleo reduzam sua dependência dos combustíveis fósseis. Os biocombustíveis renováveis, com balanço de produção energético positivo e baixa emissão de carbono (no ciclo de vida completo), constituem uma alternativa tanto para a redução da dependência do petróleo como para a mitigação dos gases do efeito estufa. O uso de bioetanol em motores SI (ignição por centelha, do inglês spark ignition), como substituto ou complemento da gasolina, vêm sendo amplamente explorado em diversos países. No Brasil e nos EUA, o etanol teve um grande crescimento na década de 1970 para suprir a crise do petróleo e apoiar o setor agrícola [3] [4], [5]. Na Europa, o etanol foi introduzido como um combustível renovável de baixo impacto de carbono e para reduzir o consumo de combustíveis fósseis [6], 
[7]. A disponibilidade de terra para o cultivo e a concorrência com o mercado agrícola ainda são fatores que influenciam na afirmação do etanol em cenários regionais. Isso resulta em diferentes estratégias sobre o uso do combustível. No Brasil, o etanol hidratado (96\% de etanol em água em uma relação volumétrica v/v) é amplamente acessível, juntamente com a gasolina comum ("gasohol" - 27\% de etanol em gasolina v/v). Nos EUA, o E10 é o principal combustível, apesar do E85 também estar disponível em alguns estados. Na Europa, as legislações exigem ao menos 5\% de etanol na gasolina desde 2013, com um futuro acréscimo para $10 \%$ nos próximos anos. Apesar disso, alguns países ainda apresentam resistência a essa mudança.

Buscando a melhor sinergia entre as propriedades do combustível e as tecnologias do motor para aumento de eficiência energética, a aplicação de estratégias não convencionais de tempos de válvula tem sido analisada em várias pesquisas. Olhando para o ciclo termodinâmico, há duas metas de interesse para aumentar a eficiência de motores SI: reduzir o trabalho de bombeamento e aumentar a eficiência de conversão de combustível. Para a redução de perdas por bombeamento, existem dois caminhos principais a serem seguidos: a utilização de combustão de mistura pobre com uma abertura maior de borboleta (maior pressão no coletor de admissão) ou a operação sem restrição de borboleta com fechamento variável da válvula de admissão. $\mathrm{O}$ aumento na eficiência termodinâmica (conversão da energia do combustível para trabalho) está diretamente relacionado com a possibilidade de atingir o mínimo avanço de ignição para máximo torque (MBT), em concordância com a razão de compressão e as propriedades do combustível. Razões de compressão maiores podem promover um aumento na eficiência termodinâmica do motor enquanto que a resistência à autoignição do combustível determinará a possibilidade de se atingir a condição MBT. A utilização de recirculação dos gases da exaustão (EGR) constitui outra alternativa que tem sido explorada para reduzir o trabalho de bombeamento e aumentar a eficiência termodinâmica, em detrimento da estabilidade de combustão em motores SI.

Esse estudo investigou o uso de etanol anidro (E100) em um motor aspirado, monocilíndrico, de 0.35 litros com sistema eletro-hidráulico de quatro válvulas. Conceitos avançados de eventos de válvula como operação sem restrição de borboleta (WOT, do inglês wide open throttle) e técnicas de contenção de gases residuais foram utilizadas para redução de perdas por bombeamento. Isso resultou em aumento de eficiência em condições de operação estequiométricas.

\section{APARATO EXPERIMENTAL}

Os experimentos foram realizados em um motor monocilíndrico de testes. Um sistema eletrohidráulico permitiu o controle independente das quatro válvulas, o qual possibilitava a operação do motor em ciclos de dois e quatro tempos. A Tabela 1 apresenta as especificações técnicas do motor e a Figura 1 exibe um esquema da célula de testes.

O motor era conectado a um dinamômetro AC, que viabilizava a condução de ensaios em modo motorizado (sem a ocorrência de combustão) e com processo de combustão ("motoring and firing tests"). A célula de testes do motor possuía um sistema de loop fechado para controle de arrefecimento e temperatura do óleo, mantendo ambos a $363 \mathrm{~K}$. Um medidor Endress+Hauser Promass 83A Coriolis foi utilizado para realizar medições vazão mássica de combustível. A vazão mássica de ar de admissão foi avaliada por um medidor de escoamento laminar Hasting 
HFM - 200. A temperatura do ar na admissão foi mantida a $303 \pm 5 \mathrm{~K}$. A pressão dentro do

Tabela 1 - Especificações técnicas do motor

\begin{tabular}{|l|l|}
\hline Modelo do motor & Ricardo Hydra Camless Two/four-stroke \\
\hline Volume deslocado & $350 \mathrm{~cm}^{3}$ \\
\hline Diâmetro & $81,6 \mathrm{~mm}$ \\
\hline Curso & $66,9 \mathrm{~mm}$ \\
\hline Razão de compressão & $11,8: 1$ \\
\hline $\begin{array}{l}\text { Câmara de } \\
\text { combustão }\end{array}$ & $\begin{array}{l}\text { Quatro válvulas pent-roof com vela de } \\
\text { ignição centralizada }\end{array}$ \\
\hline Combustível & Etanol anidro (E100) \\
\hline Injetor (DI) & $\begin{array}{l}\text { Tipo solenoide, seis furos, montado } \\
\text { lateralmente - Magneti Marelli GDI }\end{array}$ \\
\hline Injetor (PFI) & Duplo( “Twin”) spray - Bosch EV 14 \\
\hline
\end{tabular}

Figura 1 - Esquema da célula de testes

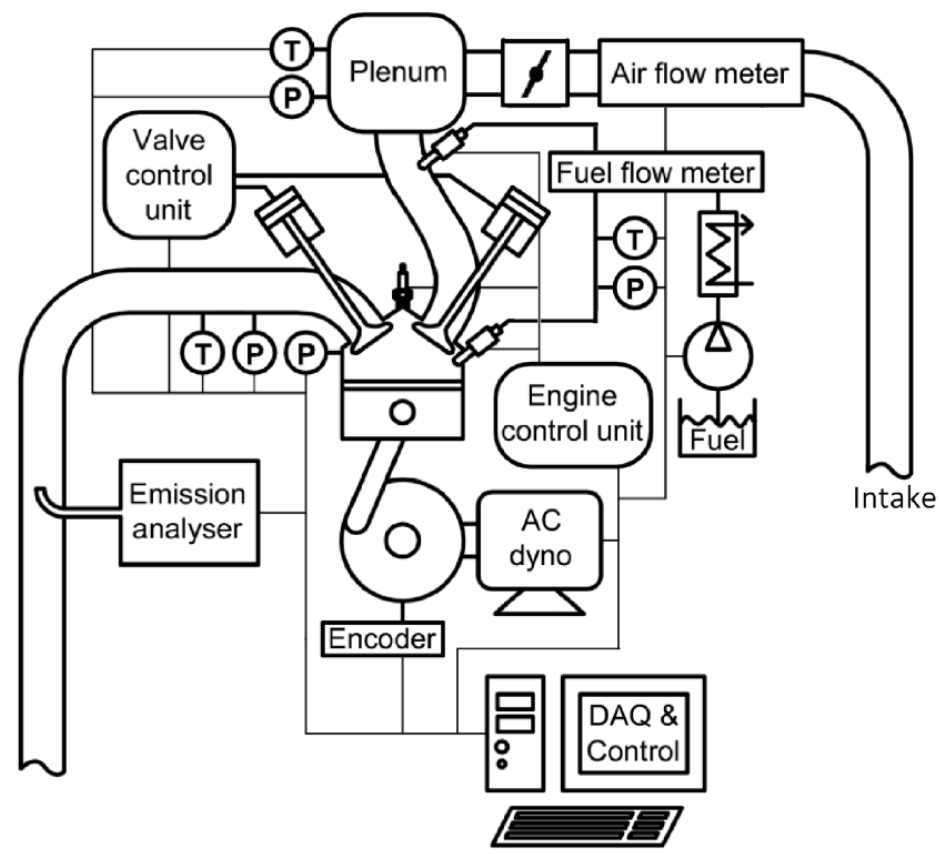

cilindro foi estimada através de um sensor de pressão piezoelétrico Kistler 6061B. A pressão na admissão foi medida por sensores piezoresistivos de pressão absoluta Kistler 4007BA20F. 
Um encoder de 720 pulsos por revolução, conectado diretamente ao virabrequim, foi utilizado para referenciar corretamente os dados obtidos aos ângulos correspondentes. Termopares do tipo $\mathrm{K}$ foram utilizados para monitorar temperaturas médias em regiões de relevância, como os dutos de admissão e exaustão, galerias de óleo e refrigeração, linha de combustível e o suprimento de óleo do sistema de válvulas. Um sistema de aquisição de dados e análise de combustão e parâmetros de operação do motor, desenvolvido internamente foi utilizado. A liberação de calor foi calculada utilizando a primeira lei da termodinâmica, como apresentado em [8].

Etanol Anidro (E100) injetado na porta de admissão (PFI) a uma pressão de 3,5 \pm 0,25 bar. Cada spray de combustível, em forma de cone, foi direcionado a uma das portas de admissão. O injetor DI foi montado lateralmente entre as válvulas de admissão e exaustão e a pressão de injeção foi mantida constante a $145 \pm 5$ bar.

Emissões de monóxido de carbono (CO), hidrocarbonetos (HC) e óxidos de nitrogênio NOx foram avaliadas utilizando um analisador Horiba MEXA-7170 DEGR. Foi aplicado um fator de correção às medições de HC, levando em conta a baixa resposta do FID (flame ionization detector) para compostos orgânicos oxigenados. Assim, a nomenclatura THC foi utilizada para se referir ao total de hidrocarbonetos, representando as emissões corrigidas de compostos orgânicos não queimados. O método de correção do FID foi apresentado em [9]-[11] enquanto que emissões específicas indicadas foram calculadas de acordo com os procedimentos apresentados em [12].

\section{METODOLOGIA}

Os ensaios foram conduzidos na rotação fixa de $1500 \mathrm{rpm}$ operando em cargas de 2.1, 3.1, 4.5, 6.1, 7.5 e 9.0 bar IMEP \pm 0.1 bar para comparar a operação convencional SI com borboleta (tSI) e a operação com os processos de NVO e ER. Ambos processos de injeção direta (DI) e port fuel injection (PFI) de etanol anidro foram explorados. Para a operação com injeção direta, foram utilizados diferentes tempos de injeção a fim de aumentar a eficiência indicada do motor. O melhor tempo de injeção observado para as estratégias ER e tSI foi em torno de 330 CAD BTDC da combustão. O melhor tempo de injeção para os casos com NVO ocorreu durante a fase inicial do período de recompressão. A injeção indireta (PFI) nas portas de admissão foi configurada para o TDC da compressão para propiciar um período maior de preparação da mistura ar-combustível.

Nos ensaios convencionais tSI, as durações dos eventos de válvulas (admissão e exaustão) foram mantidas constantes. A restrição por borboleta foi utilizada para reduzir a densidade do ar e promover o controle da carga.

Para a estratégia NVO [13]-[16] o momento de fechamento da válvula de exaustão foi adiantado (EIVC, do inglês early intake valve closure) para conter parte do gás residual no cilindro. Esse término precoce da exaustão resulta na recompressão dos gases residuais. A fim de reduzir o trabalho por bombeamento, a abertura da válvula de admissão foi atrasada em relação ao TDC (quase que simetricamente). Assim, parte do trabalho de compressão pôde ser recuperado na forma de trabalho de expansão. O fechamento da válvula de admissão foi adiantado para o momento em que suficiente mistura ar-combustível estava contida dentro do cilindro para produzir a carga do motor desejada. Com o propósito de alcançar uma boa relação entre eficiência indicada e eficiência de combustão, um estudo de otimização sobre o período 
de NVO foi previamente conduzido para todas as cargas ensaiadas. A injeção direta foi avaliada nos períodos optimizados de NVO [17]-[19].

Para os ensaios ER [20], apenas uma das válvulas de exaustão foi utilizada durante o processo de exaustão. A segunda válvula foi utilizada apenas durante o período da admissão para efetuar a re-indução de gases de escape no cilindro. Esse procedimento se fez necessário devido a limitações do hardware de gerenciamento de eventos de válvulas, o qual não possibilitava a abertura de ambas as válvulas duas vezes por ciclo. O início do período de ER e sua duração variaram de acordo com a carga e a estratégia de injeção para atingir uma eficiência indicada otimizada. Operação em regime HCCI não foi priorizada conforme [21]-[23]. O evento de abertura de válvula de admissão (IVO) foi configurado próximo ao TDC e o fechamento da válvula de admissão (IVC) foi configurado conforme a carga necessária.

\section{RESULTADOS}

\subsection{Processo de trocas gasosas ("gas exchange process")}

A figura 2 apresenta a pressão no coletor de admissão e a pressão média efetiva de bombeamento (PMEP) para as três estratégias de tempos de válvulas em diferentes cargas. A operação sem restrição de borboleta com método de controle de carga através de fechamento adiantado da válvula de admissão possibilitou operação com pressão de coletor próxima à atmosférica. O fechamento da válvula de admissão (IVC) ocorreu nos pontos em que havia quantidade suficiente de ar fresco para promover a combustão estequiométrica na carga desejada. No caso onde o controle de carga era realizado pela restrição de ar aspirado pela borboleta, a pressão do pleno foi reduzida para diminuir a densidade da mistura. A injeção de combustível nas portas de admissão deslocou ar de admissão exigindo pressão de admissão maior para a mesma carga de operação, comparando os casos PFI com DI. Entretanto, a operação com injeção direta intensificou os efeitos de resfriamento dentro do cilindro, o que resultou em pressões menores previamente ao início da compressão. A operação em plena carga foi diretamente afetada pelo deslocamento de ar e pelos efeitos de resfriamento da carga dento do cilindro. Por esses motivos, cargas máximas de 10.4 bar e 9.9 bar IMEP foram encontradas para as estratégias de injeção DI e PFI, respectivamente.

A pressão menor no coletor com operação tSI aumentou o trabalho de bombeamento, o qual é a principal causa da baixa eficiência de motores SI em carga parcial. No caso com NVO, os gases da exaustão recomprimidos (como consequência do fechamento precoce da válvula de exaustão) se expandiram até aproximadamente a pressão atmosférica quando a válvula de admissão era aberta. A pressão dentro do cilindro foi mantida em torno do nível atmosférico até o fechamento da válvula de admissão (IVC), a partir de quando uma fase de sobre-expansão ocorria. O trabalho consumido na fase de sobre-expansão era parcialmente recuperado durante o ciclo de potência.

Na estratégia ER diferentes períodos de re-indução (rebreathing) de gases de escape resultaram em comportamentos distintos de PMEP (pressão média efetiva de bombeamento, do inglês pumping mean effective pressure). Para 2.0 e 3.1. bar IMEP o período de ER foi curto e seu melhor posicionamento ocorreu durante o intervalo de sobreposição com a válvula de admissão. Como na estratégia NVO, IVC foi utilizado como estratégia de controle de carga. Entre 4.5 bar IMEP e 9 bar IMEP um período separado de re-indução, com a segunda válvula de exaustão 
Figura 2 - Pressão da admissão e PMEP para as diferentes estratégias de acionamento de válvulas em várias cargas do motor
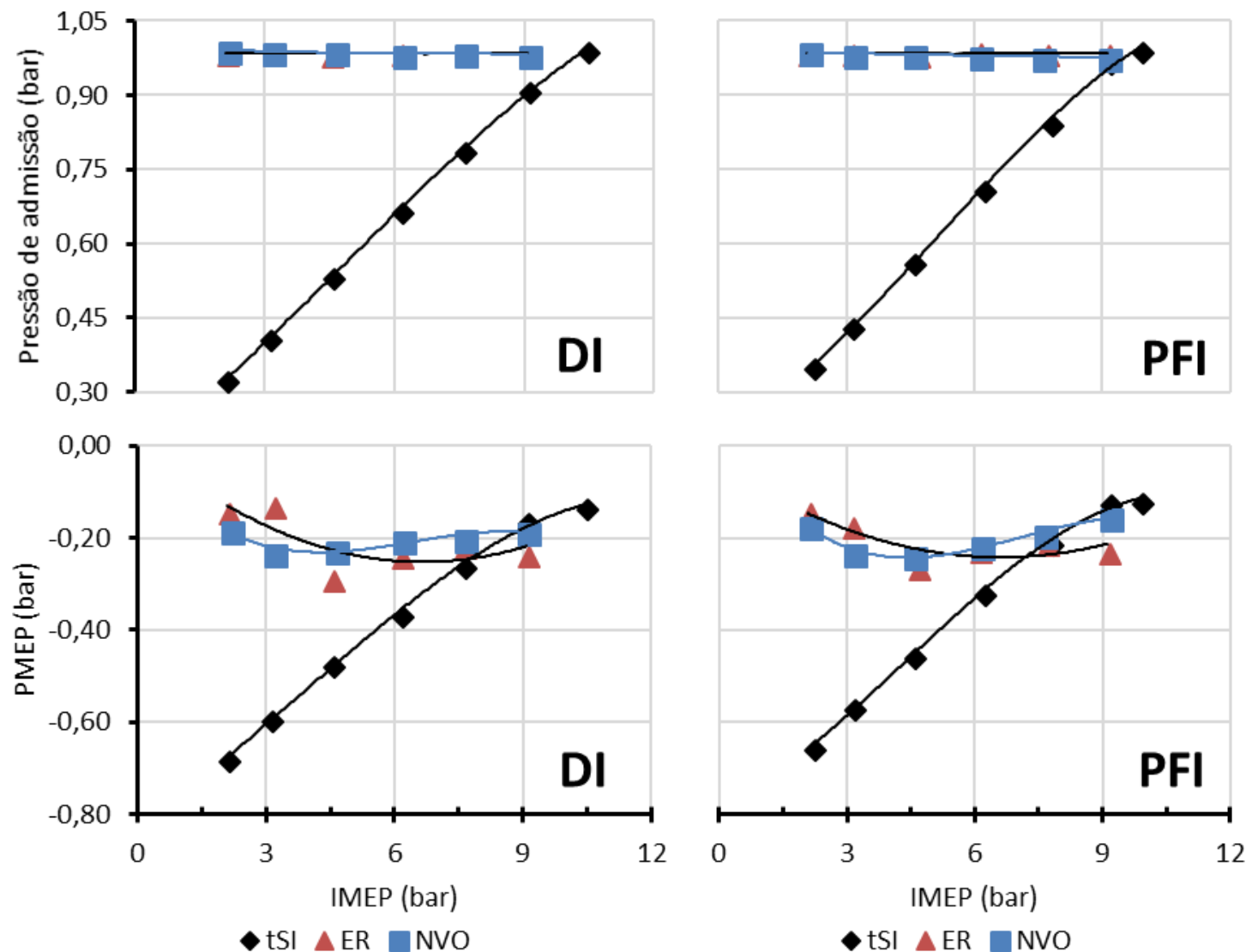

abrindo perto do IVC, resultou na melhor eficiência indicada. A 4.5 bar IMEP a melhor eficiência ocorreu ao se fechar a válvula de exaustão ao redor do BDC. Para as cargas maiores, EVC ocorreu relativamente depois de BDC. Verificou-se um aumento no trabalho de bombeamento da exaustão em cargas maiores devido a atuação de apenas uma válvula durante o processo de exaustão. Esse fato provocou aumento de PMEP (valores mais negativos) quando comparados os métodos de ER com NVO.

\subsection{Análise da combustão}

A figura 3 apresenta o tempo de ignição e o ângulo referente ao momento em que $50 \%$ da carga inicial foi queimada (CA50), ambos para MBT. Além disso, apresenta a duração da combustão

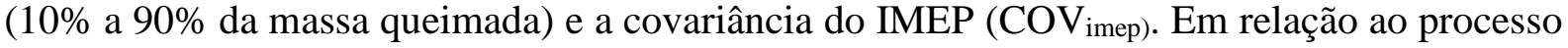
da combustão, o aumento na fração de gases residuais (RGF) quente exigiu tempos de ignição mais avançados para determinar a combustão corretamente. Como a estratégia de controle de carga EIVC foi empregada, reduções das estruturas de grande escala do escoamento (swirl e tumble) dentro do cilindro gerou redução dos níveis de turbulência pré-ignição. Isso resultou em maiores durações de combustão. Não foi possível alcançar ótimo posicionamento de ignição nos casos de baixa carga, mesmo com maiores avanços de ignição. Nesses pontos, o avanço de ignição resultou em falha de ignição e perda da combustão. O maior avanço de ignição dos casos de ER foi necessário devido às maiores frações de RGF. Como os tempos de acionamento 
Figura 3 - Parâmetros de combustão para as diferentes estratégias de acionamento de válvulas em várias cargas do motor
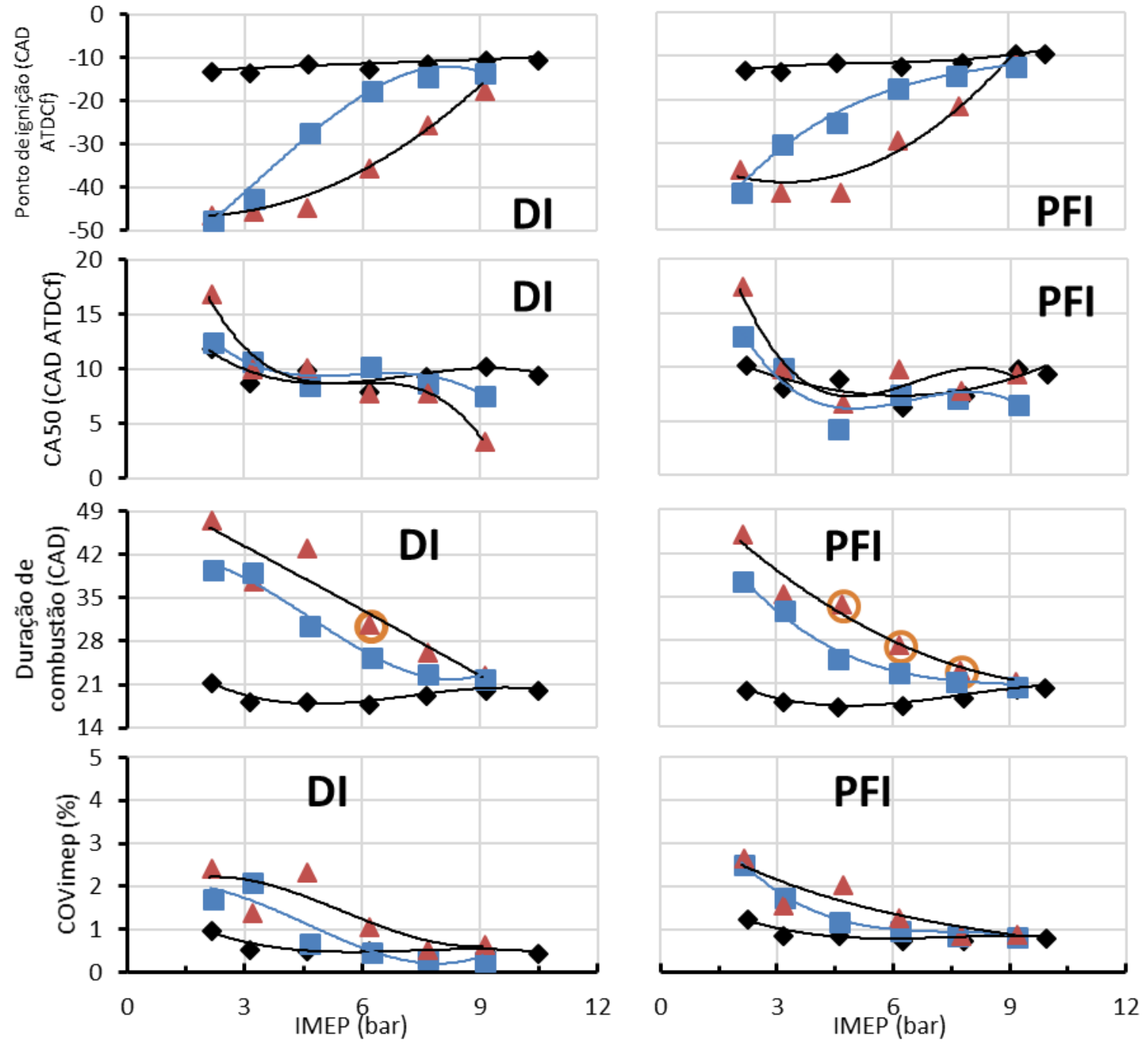

das válvulas foram levemente diferentes para as estratégias de DI e PFI, houve uma pequena diferença nos tempos de ignição. Em geral, a menor temperatura de compressão anterior à ignição nos casos DI exigiu um avanço de ignição maior, como consequência do efeito de arrefecimento da injeção direta. Isso também se refletiu na duração da combustão (10\% a 90\% da fração de massa queimada), que tende a ser mais rápida nos casos de PFI devido às temperaturas maiores de combustão e à maior homogeneidade da carga.

A estratégia de ER resultou nas maiores durações de combustão devido às maiores frações de RGF, seguida pela estratégia NVO. Nos casos tSI a combustão ocorreu predominantemente com propagação de chama sem ocorrência de detonação. Para a estratégia NVO, os pontos otimizados resultaram em uma combustão por deflagração de chama turbulenta pré-misturada convencional de motores SI. Porém, apresentaram uma maior duração de combustão resultante da maior quantidade de RGF. Além disso, a estratégia NVO resultou em uma menor razão de compressão efetiva e baixos níveis de turbulência (esperados) dentro do cilindro. Para alguns dos pontos de NVO testados (durante a fase de optimização da estratégia de NVO), foi possível obter ignição por compressão auxiliada por centelha (SACI, do inglês spark assisted compression ignition), operando com injeção do tipo PFI. Utilizando a estratégia ER e a injeção 
do tipo PFI, a taxa de quantidade de calor liberado indicou combustão SACI para diversas cargas. Já com o uso de DI, para a estratégia ER, a combustão SACI ocorreu apenas para a carga de 6.1 bar de IMEP. Os pontos referentes à combustão SACI estão circulados no gráfico relacionado à duração da combustão na Figura 3.

O maior tempo de duração da combustão influenciou no acréscimo da variabilidade cíclica da combustão. Nos casos de ER, a maior complexidade do processo de trocas gasosas contribuiu para o incremento da $\mathrm{COV}_{\text {imep. }}$

Mesmo tendo ocorrido um processo de combustão mais longo com as estratégias de NVO e ER, o pico de pressão no cilindro (Figura 4) se manteve aproximadamente aos mesmos níveis (para as mesmas cargas do motor). A massa de ar acrescentada à mistura no cilindro, devido à adição de RGF, foi responsável pelo acréscimo de pressão em temperaturas menores de combustão. Ambos os tipos de injeção, PFI e DI, apresentaram picos de pressão no cilindro similares. No caso de utilização de ER com DI, a taxa de aumento de pressão foi mantida em níveis aceitáveis, com um máximo de 3,7 bar/CAD em uma carga do motor de 9.0 bar de IMEP.

A temperatura de exaustão (Figura 4) mostrou diferentes tendências de acordo com os tempos de válvula e estratégias de injeção. Uma aceitável eficiência de conversão de CO/HC/NOx seria esperada ao se utilizar um catalisador convencional de três vias em operação estequiométrica nessas condições de temperatura.

Figura 4 - Pressão máxima no cilindro e temperatura de exaustão para as diferentes estratégias de acionamento de válvulas em várias cargas do motor
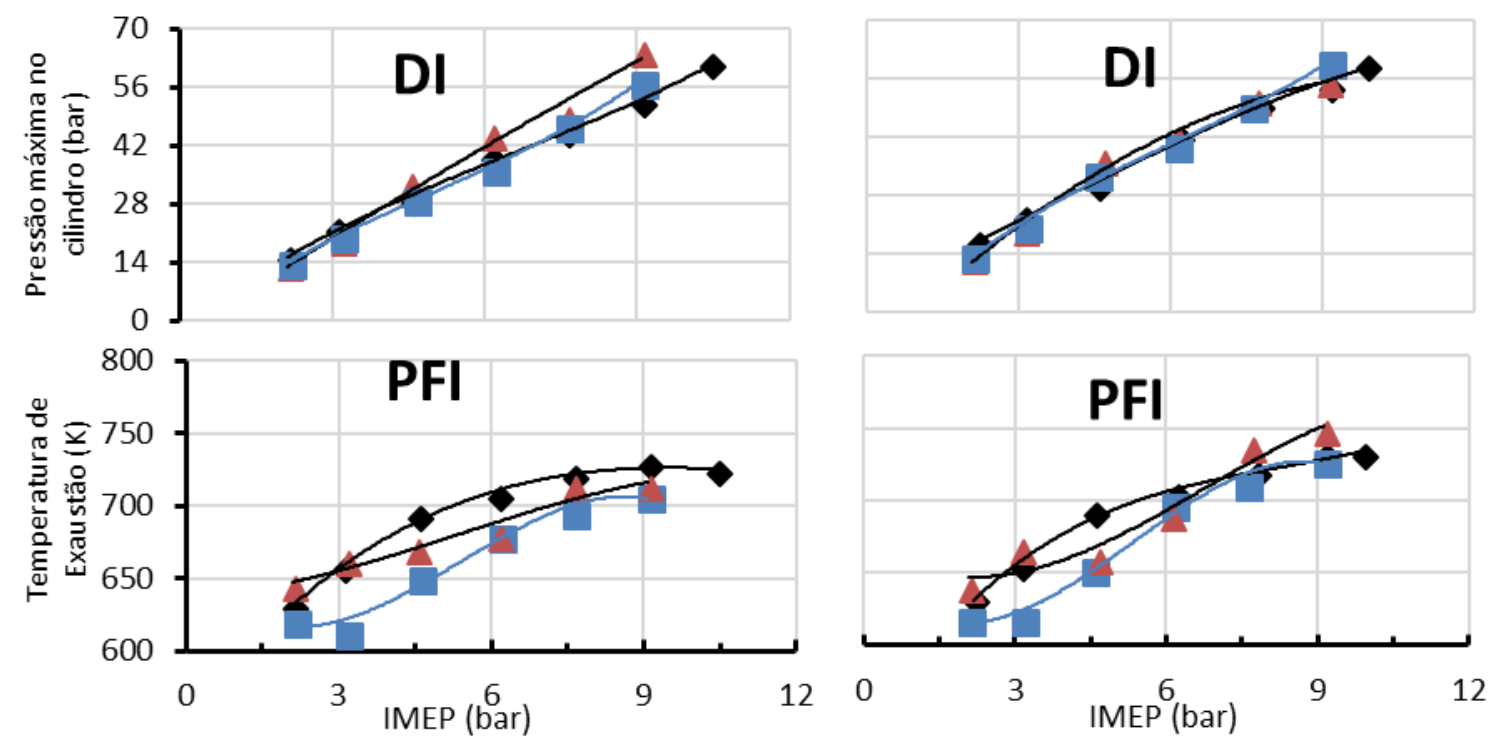

\subsection{Emissões específicas}

As emissões específicas indicadas de $\mathrm{CO}, \mathrm{THC}_{\mathrm{e}} \mathrm{NO}_{\mathrm{x}}$ estão apresentadas na figura 5. Verificouse que a menor formação de CO ocorreu na estratégia de NVO, seguida de ER e tSI. A condição de NVO com injeção adiantada durante o período de recompressão propiciou o maior tempo para a mistura da carga entre todos os casos de injeção direta. Como a formação de CO está diretamente ligada a homogeneização da mistura, essa estratégia promoveu a menor formação de CO. Entretanto, o caso tSI com injeção direta durante a fase inicial da admissão, apresentou 
redução na homogeneidade dentro do cilindro a medida em que a carga do motor aumentou. Para os casos ER, houve uma relação direta entre a redução de CO e o aumento de THC. Essa relação ocorreu devido à baixa temperatura de combustão, a qual não foi suficiente para oxidar parcialmente o combustível. No caso PFI, foram observados índices menores de emissões de CO quando comparado com as condições de injeção direta devido ao maior período para homogeneização da carga na porta de admissão.

A tendência de THC de cada estratégia demonstrou similaridade para ambos os casos PFI e DI. Contudo, as emissões de THC tenderam a ser menores na operação PFI devido à maiores temperaturas de combustão. Porém isto resultou em maior formação de $\mathrm{NO}_{\mathrm{x}}$ para os casos em que foi utilizado PFI.

Na comparação entre as diferentes estratégias de tempos de válvulas em cargas médias no motor, a estratégia ER apresentou níveis maiores de RGF, ocasionando temperaturas de combustão menores e consequentemente reduzindo emissões de $\mathrm{NO}_{\mathrm{x}}$. Devido à injeção direta adiantada durante a fase de recompressão o processo de vaporização do combustível reduziu a temperatura do RGF. Isso propiciou redução de temperatura pré-ignição e consequentemente reduzindo a temperatura de combustão. Como resultado, os casos NVO com DI produziu menos $\mathrm{NO}_{\mathrm{x}}$ quando comparado aos casos NVO com PFI.

Figura 5 - Emissões do motor para as diferentes estratégias de acionamento de válvulas em várias cargas do motor
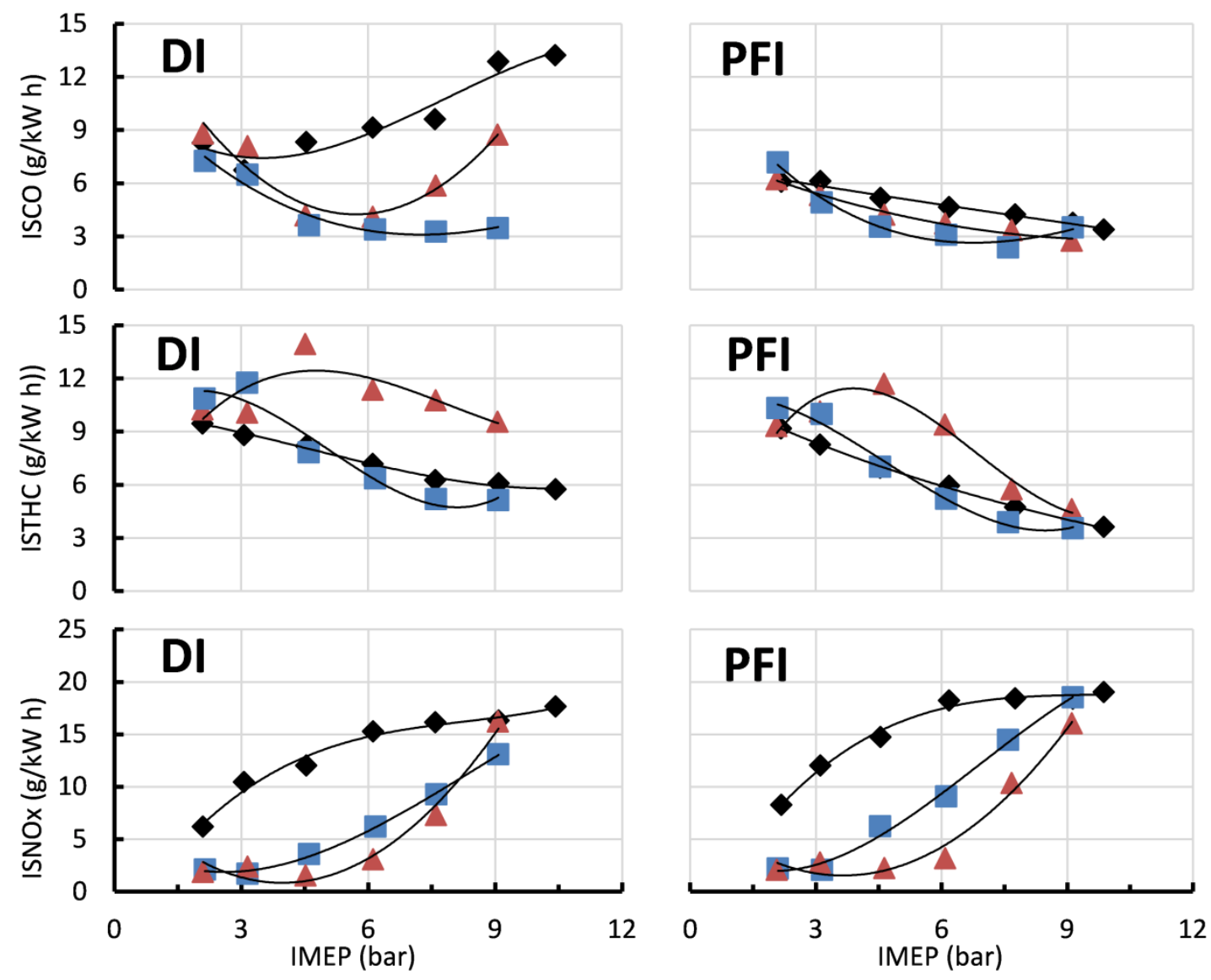

$\diamond \mathrm{tSI} \triangle \mathrm{ER} \square \mathrm{NVO}$ 


\subsection{Parâmetros de eficiência}

A pressão média efetiva de bombeamento (PMEP) foi um fator determinante na eficiência de trocas gasosas, que correlaciona a quantidade de trabalho consumido durante as fases de transferência de gases ao trabalho produzido durante o ciclo de potência. Os valores mais expressivos de PMEP, causados pela estratégia tSI, afetaram a eficiência de trocas gasosas. Maiores eficiências foram observadas sob as condições PFI. As estratégias ER e NVO apresentaram eficiências similares. Porém, devido ao fechamento adiantado da válvula de admissão (EIVC) no caso NVO, maior quantidade de energia pôde ser recuperada durante o ciclo de potência. Com o motor operando em cargas inferiores a 4.0 bar IMEP, o processo de exaustão restrito (ocasionado pela atuação de apenas uma válvula no ciclo de exustão) reduziu a eficiência de transferência de gases no caso ER em comparação com a estratégia NVO.

Figura 6 - Parâmetros relacionados a eficiência para as diferentes estratégias de acionamento de válvulas em várias cargas do motor
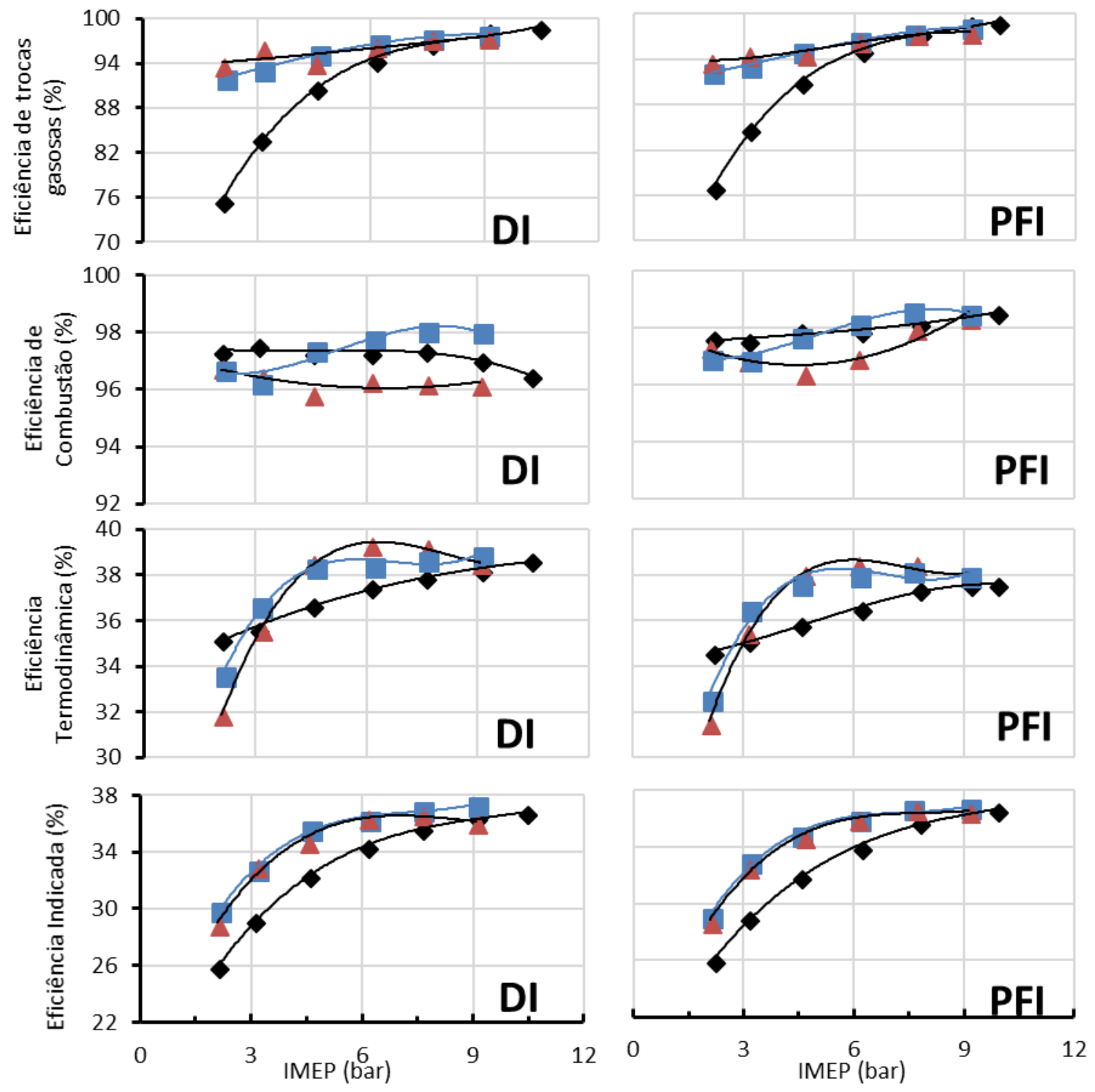
A eficiência de combustão é resultado direto das emissões de $\mathrm{CO}$ e THC, devido a sua relação com a fração de energia do combustível disponível na exaustão. Os baixos níveis de emissões observados nas estratégias com PFI resultaram em maior eficiência de combustão quando comparadas às estratégias com injeção direta. Verificou-se baixa eficiência de combustão nos casos com ER devido aos níveis elevados de emissões de THC. Por outro lado, a melhor homogeneidade da carga na estratégia NVO propiciou níveis de eficiência de combustão maiores em comparação ao caso tSI. A posterior redução da eficiência de combustão no caso tSI com injeção direta foi consequência do aumento da estratificação da carga.

A estratégia ER com combustão SACI apresentou a melhor eficiência termodinâmica em carga parcial. Isto poderia ser explicado pela baixa transferência de calor dentro do cilindro provocada pelas baixas temperaturas de combustão. Com o aumento da carga no motor as perdas por calor também aumentaram. Comparando os sistemas de preparação de mistura, DI e PFI, o efeito de resfriamento da injeção direta reduziu a temperatura dentro do cilindro e também reduziu as perdas de calor dentro do cilindro. Em baixa carga, NVO demonstrou maior potencial para aumentar a eficiência do motor.

Enfim, a eficiência indicada é um compromisso entre a eficiência de troca gasosa e a eficiência de combustão com a eficiência termodinâmica. Logo, a baixa eficiência em carga parcial da condição tSI pode ser explicada pelas baixas eficiências de troca gasosa e termodinâmica. A primeira foi comprometida devido ao controle de carga por restrição de borboleta enquanto que a segunda foi resultado das maiores perdas de calor durante a combustão com temperatura elevada. Apesar da eficiência termodinâmica da estratégia com NVO nem sempre ser a maior, o aumento na eficiência de combustão em carga média resultou em eficiência indicada relativamente maior para todas as cargas testadas, em comparação ao uso de ER. Comparando os métodos de preparação de mistura, DI e PFI, a eficiência indicada observada para a operação com injeção direta foi relativamente maior por consequência da maior eficiência termodinâmica.

\section{CONCLUSÃO}

Algumas conclusões principais podem ser destacadas: (1) ao utilizar a estratégia de NVO, a injeção direta durante o processo de recompressão promoveu uma ótima mistura da carga na câmara de combustão. Por essa razão, foi possível realizar uma comparação das eficiências de combustão com os casos de injeção do tipo PFI; (2) um processo melhor de combustão ocorreu ao se utilizar as estratégias de NVO e ER; (3) o processo intensificado de combustão com SACI resultou em maior eficiência termodinâmica; (4) a redução do trabalho de bombeamento devido ao controle de carga pelo fechamento da válvula de admissão foi de grande importância para aprimorar a eficiência do motor SI operando em carga parcial.

Portanto, as estratégias de NVO e ER apresentaram um potencial similar de aprimoramento considerável de eficiência de um motor SI naturalmente aspirado, operando com uma razão ar/combustível estequiométrica. O maior desafio na aplicação dessas estratégias de controle de válvulas poderá ser a relação custo-efetividade de sistemas de controle de válvulas mais complexos.

\section{REFERÊNCIAS}

[1] European Commission, "EU Transport in Figures," 2014. 
[2] US EPA, "DRAFT Inventory of U . S . Greenhouse Gas Emissions and Sinks : 1990 1998," 2016.

[3] J. A. DUFFIELD, I. M. XIARCHOS, and S. A. HALBROOK, "ETHANOL POLICY. PAST, PRESENT, AND FUTURE," SdL Rev, vol. 53, p. 425, 2008.

[4] F. Rosillo-Calle and L. A. B. Cortez, "Towards ProAlcool II-a review of the Brazilian bioethanol programme," Biomass and Bioenergy, vol. 14, no. 2, pp. 115-124, Mar. 1998.

[5] C. R. Soccol et al., "Brazilian biofuel program: An overview," J. Sci. Ind. Res. (India)., vol. 64, no. 11, pp. 897-904, 2005.

[6] European Parliament, "Directive 2009/28/EC of the European Parliament and of the Council of 23 April 2009," Off. J. Eur. Union, vol. 140, no. 16, pp. 16-62, 2009.

[7] European Union, "Directive 2009/30/EC of the European Parliament and of the Council," Off. J. Eur. Union, no. April, p. L140/88-L140/113, 2009.

[8] J. B. Heywood, Internal Combustion Engine Fundementals, vol. 21. 1988.

[9] K. Kar, R. Tharp, M. Radovanovic, I. Dimou, and W. K. Cheng, "Organic gas emissions from a stoichiometric direct injection spark ignition engine operating on ethanol/gasoline blends," Int. J. Engine Res., vol. 11, no. 6, pp. 499-513, Dec. 2010.

[10] T. Wallner, "Correlation Between Speciated Hydrocarbon Emissions and Flame Ionization Detector Response for Gasoline/Alcohol Blends," J. Eng. Gas Turbines Power, vol. 133, no. 8, p. 082801, 2011.

[11] T. D. M. Lanzanova, M. Dalla Nora, and H. Zhao, "Performance and economic analysis of a direct injection spark ignition engine fueled with wet ethanol," Appl. Energy, vol. 169, pp. 230-239, May 2016.

[12] Economic Commission for Europe of the United Nations, "Regulation No 49 of the Economic Commission for Europe of the United Nations (UN/ECE)," Off. J. Eur. Union, no. 171, pp. 1-390, 2013.

[13] D. Law, D. Kemp, J. Allen, G. Kirkpatrick, and T. Copland, "Controlled Combustion in an IC-Engine with a Fully Variable Valve Train," SAE Tech. Pap., no. 2001-1-251, 2001.

[14] L. Koopmans and I. Denbratt, "A four-stroke camless engine, operated in homogeneous charge compression ignition mode with commercial gasoline," SAE Tech. Pap., no. 2001-01-3610, 2001.

[15] H. Zhao, J. Li, T. Ma, and N. Ladommatos, "Performance and analysis of a 4-stroke multi-cylinder gasoline engine with CAI combustion," no. 2002-1-420, 2002.

[16] Y. Zhang, B.-Q. He, H. Xie, and H. Zhao, "The Combustion and Emission Characteristics of Ethanol on a Port Fuel Injection HCCI Engine," SAE Tech. Pap., no. 2006-1-631, 2006.

[17] T. Urushihara, K. Hiraya, A. Kakuhou, and T. Itoh, "Expansion of HCCI operating region by the combination of direct fuel injection, negative valve overlap and internal fuel reformation," SAE Tech. Pap., no. 10.4271/2003-01-0749, 2003.

[18] G. Gnanam, M. Johnson, A. Sobiesiak, and G. Reader, "HCCI Combustion With Internal Fuel Reforming, Varied Levels of EGR and Charge Preheat - A Computational Study," SAE Tech. Pap., no. 2005-1-140, 2005.

[19] M. Tongroon and H. Zhao, "Analysis of the effect of direct injection of alcohol fuel on minor heat release reactions and controlled autoignition combustion," Proc. Inst. Mech. Eng. Part D J. Automob. Eng., vol. 226, no. 12, pp. 1678-1688, May 2012.

[20] H. Zhao, HCCI and CAI engines for the automotive industry. Woodhead Pub., 2007.

[21] A. Fuerhapter, W. F. Piock, and G. K. Fraidl, "CSI - Controlled Auto Ignition - the Best Solution for the Fuel Consumption - Versus Emission Trade-Off?," SAE Tech. Pap., no. 2003-1-754, 2003. 
[22] a Fuerhapter, E. Unger, W. F. Piock, and G. K. Fraidl, "The new AVL CSI Engine HCCI Operation on a Multi Cylinder Gasoline Engine," SAE Tech. Pap., no. 2004-1$551,2004$.

[23] F. Duffour, F. Vangraefschèpe, V. Knop, and L. De Francqueville, "Influence of the Valve-lift Strategy in a CAI ${ }^{\mathrm{TM}}$ Engine using Exhaust Gas Re-Breathing - Part 1: Experimental Results and 0D Analysis," SAE Tech. Pap., no. 2009-1-299, 2009. 